\title{
Reconstructing air showers with LOFAR using event specific GDAS atmospheres
}

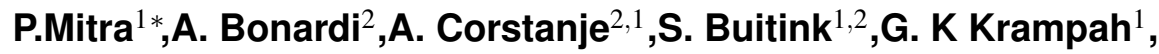 \\ H. Falcke ${ }^{2,3,4}$,B. M Hare ${ }^{5}$, J. R. Hörandel ${ }^{1,2,3}$, T. Huege ${ }^{1,8}$, \\ K. Mulrey ${ }^{1}$,A Nelles ${ }^{6,9}$,H Pandya ${ }^{1}$,J.P. Rachen ${ }^{2,1}$,L. Rossetto ${ }^{2}$, \\ O. Scholten ${ }^{5,7}$,S. ter Veen ${ }^{2}$,T.N.G. Trinh ${ }^{5,10}$,T. Winchen ${ }^{1}$
}

${ }^{1}$ Astrophysical Institute, Vrije Universiteit Brussel, Pleinlaan 2, 1050 Brussels, Belgium

${ }^{2}$ Department of Astrophysics / IMAPP, Radboud University Nijmegen, P. O. Box 9010, 6500 GL, Nijmegen, The Netherlands

${ }^{3}$ NIKHEF, Science Park Amsterdam, 1098 XG Amsterdam, The Netherlands

${ }^{4}$ Netherlands Institute of Radio Astronomy (ASTRON), Postbus 2, 7990 AA Dwingeloo, The Netherlands

${ }^{5}$ KVI-CART, University Groningen, P. O. Box 72, 9700 AB Groningen, The Netherlands

${ }^{6}$ DESY, Platanenallee 6, 15738 Zeuthen, Germany

${ }^{7}$ Interuniversity Institute for High-Energy, Vrije Universiteit Brussel, Pleinlaan 2, 1050

Brussels, Belgium

${ }^{8}$ Institut für Kernphysik, Karlsruhe Institute of Technology(KIT), P. O. Box 3640, 76021, Karlsruhe, Germany

${ }^{9}$ ECAP, Friedrich-Alexander-University Erlangen-Nürnberg, 91058 Erlangen, Germany

${ }^{10}$ Department of Physics, School of Education, Can Tho University Campus II, 3/2 Street, Ninh Kieu District, Can Tho City, Vietnam

E-mail: pmitra@vub.be

Estimating the depth of shower maximum $\mathrm{X}_{\max }$ with high precision is of great interest for the study of primary particle composition. One of the systematic uncertainties in reconstructing $X_{\max }$ from the radio emission of air showers is the limited knowledge of the atmospheric parameters like humidity, pressure, temperature and the index-of refraction. Using the Global Data Assimilation System (GDAS), a global atmospheric model, we have implemented time-dependent realistic atmospheric profiles in the air shower simulation codes CORSIKA and the radio plug-in CoREAS. This program is now available within CORSIKA and flexible to be adapted for different air shower experiments. We have analyzed the LOFAR cosmic ray data with dedicated simulations for each detected air shower with event specific GDAS atmospheres and investigated the effects of pressure and humidity on the reconstructed $X_{\max }$. This study shows that for bulk of the events, where the ground pressure is close to US standard atmosphere values, there is a small systematic shift in $X_{\max }$ that is less than $2 \mathrm{~g} / \mathrm{cm}^{2}$ and for very low pressure values the shift is up to $15 \mathrm{~g} / \mathrm{cm}^{2}$.

36th International Cosmic Ray Conference -ICRC2019-

July 24th - August 1st, 2019

Madison, WI, U.S.A.

* Speaker. 


\section{Introduction}

The radio detection of cosmic rays is a promising technique providing deeper insight in the development of extensive air showers. Estimating the depth of shower maximum $\mathrm{X}_{\max }$ with improved accuracy is of great interest for the study of the primary particle composition [1]. Thus, the knowledge of atmospheric variables like temperature, humidity and pressure are important for the estimation of $X_{\max }$. The highest precision of the measurement of $X_{\max }$ with the radio technique has been currently achieved with the LOFAR radio telescope, situated in the north of the Netherlands. The dense core of LOFAR consists of 288 low-band antennas within a diameter of 320 meters, recording cosmic ray events in the $30-80 \mathrm{MHz}$ band. This provides the opportunity to investigate the radio footprint, i.e.the lateral intensity distribution in close detail and enables us to infer $\mathrm{X}_{\max }$ to a precision of $20 \mathrm{~g} / \mathrm{cm}^{2}$ which is comparable to the precision achieved with the fluorescence detectors [2]. The precision of the reconstruction is sensitive to the choice of an atmospheric model included in the Monte Carlo air shower simulation codes. The measured lateral intensity distributions are compared to CoREAS [3], a simulation package for the radio emission from the individual particles in the cascade simulated with CORSIKA . There are several options for including parameterized atmospheres for different observatories [4]. The refractive index of air also plays a crucial role in the radio emission of cosmic rays. It determines the propagation velocity of the radio signal at different altitudes and causes highly time-compressed signals on ground for observers located at the corresponding Cherenkov angle. The angle for Cherenkov emission is sensitive to the refractive index $n$. Thus variations in the refractive index lead to changes in the radio intensity footprint on the ground [5]. Therefore, a realistic description of the atmospheric density and refractive index profiles needs to be incorporated in CoREAS. In LOFAR analysis, dedicated simulation sets for each detected shower are used. This requires that atmospheric profiles be included in the simulation which closely matches the conditions during the time of actual measurements for an accurate estimation of $\mathrm{X}_{\max }$.

We report the results of a study on the effects of atmospheric parameters like pressure and humidity on the reconstructed $X_{\max }$ analyzing LOFAR data,by including event-specific atmospheric conditions using GDAS, a global meteorological model. For this purpose we have developed a program that extracts GDAS atmospheric parameters which is interfaced with CORSIKA. This program is available for public use since the release of CORSIKA version 7.6300. It is flexible and can be adapted by the users to obtain parameterized atmospheric profiles for user-specified times and locations.

\section{Atmopsheric profiles from GDAS}

The Global Data Assimilation System (GDAS) is a database of atmospheric data used for weather forecasting [6]. It provides data, every three hours, for several atmospheric state variables packed in $1^{\circ} \times 1^{\circ}$ latitude longitude grid. For air shower analysis important parameters are temperature, pressure, relative humidity, air density, and atmospheric depth at several altitude levels. The first three quantities and the altitude are directly available in the GDAS data and are used to calculate air density and atmospheric depth. Next, the refractivity $N$ defined as $N=(n-1) 10^{6}$ can 
be expressed as as a function of humidity, pressure and temperature[5]

$$
N=77.6890 \mathrm{KhPa}^{-1} \frac{p_{d}}{T}+71.2952 \mathrm{KhPa}^{-1} \frac{p_{w}}{T}+375463 \mathrm{~K}^{2} \mathrm{hPa}^{-1} \frac{p_{w}}{T^{2}}
$$

with $p_{w}, p_{d}$ and $T$ being the partial water vapor pressure, partial dry air pressure and temperature respectively. The effect of humidity is important for our study as it tends to increase the refractivity in comparison to that of dry air in the radio frequency regimes. We studied several atmospheric profiles at LOFAR. Fig.1 (left) shows the mean profile for the relative difference in refractivity $\Delta N_{\text {relative }}$ between GDAS and the US standard atmosphere as a function of altitude for over 3 years consisting of 100 atmospheric profiles that correspond to the time of recorded cosmic ray events at LOFAR. It is defined as $\Delta N_{\text {relative }} \%=\frac{N_{\mathrm{GDAS}}-N_{\mathrm{US}}}{N_{\mathrm{US}}} \times 100, N_{\mathrm{GDAS}}$ is calculated from Eq-2.1 using GDAS atmospheres at LOFAR and $N_{\mathrm{US}}$ is obtained from a linear scaling of the density profile using US standard atmosphere with respect to a fixed ground level refractivity. The absolute value of the mean $\Delta N_{\text {relative }}$ is around $10 \%$ near ground and around $3-8 \%$ between 3 and $10 \mathrm{~km}$ of altitude, the region important for shower development. To account for the propagation effects of the radio signal, it is important to calculate the effective integrated refractive index between the point of emission and the observer. The typical values for relative effective refractive indices are around 7-10\% from the altitude range $3-10 \mathrm{~km}$. Fig.1(right) shows the difference in atmospheric depth profile between the US standard atmosphere and the GDAS atmospheres at LOFAR for 8 profiles over the years 2011 - 2016. The GDAS atmospheres vary significantly from the US atmosphere. Atmospheric profiles with similar ground atmospheric depth can evolve differently higher in the atmosphere. This is important for calculating the correct geometric distance to the shower maximum.
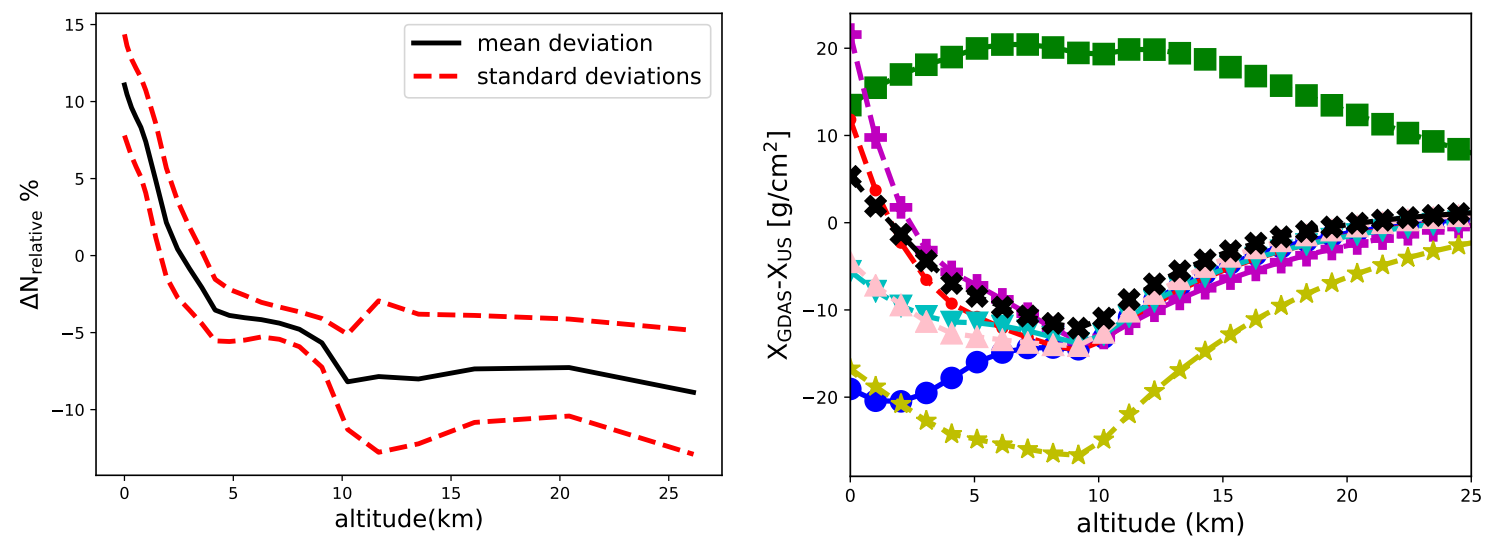

Figure 1: Left: Mean relative refractivity as a function of altitude for 100 atmospheric profiles at LOFAR spanning over the years 2011 to 2014. The black solid line denotes the mean profile and the red dashed lines show the standard deviations. Right: profiles for the difference in atmospheric depth between US standard atmosphere and GDAS atmospheres at LOFAR as a function of altitude between the years $2011-2016$.

\section{Implementation of GDAS in CORSIKA/CoREAS}

We have developed a program called 'gdastool'that extracts the required GDAS atmospheric parameters given the coordinates of an observatory and UTC timestamp of the event. It then returns 
the parameterized density profile fitted with the layered atmospheric model used in CORSIKA with proper boundary conditions and additionally a table for refractive index including humidity effects as a function of height in $1 \mathrm{~m}$ step size. The option for GDAS parameterized atmosphere can be invoked by a new keyword 'ATMF'in the CORSIKA input file. This feature is available as of CORSIKA version 76300 .

\section{Results and analysis}

We have analyzed LOFAR events simulated with new GDAS atmospheres and compared the reconstructed $\mathrm{X}_{\max }$ [2] between various simulation sets

- Set A-the showers were simulated with CORSIKA v-7.6300 and GDAS atmosphere including humidity effects.

- Set B-the showers were simulated with CORSIKA v-7.4385 and US standard atmosphere.

- Set $\mathbf{C}$-this set is identical to Set B but with an additional correction factor to correct for realistic atmospheres posterior to the reconstruction. This is done by reconstructing the geometrical distance to the shower maximum using CoREAS simulations with US standard atmospheres, and then calculating the corresponding value for using a realistic GDAS atmosphere. This correction compensates for changes in the total mass overburden, but not $X_{\max }$ for effects related to changes in the index of refraction. This method was used in previous LOFAR analysis [2].

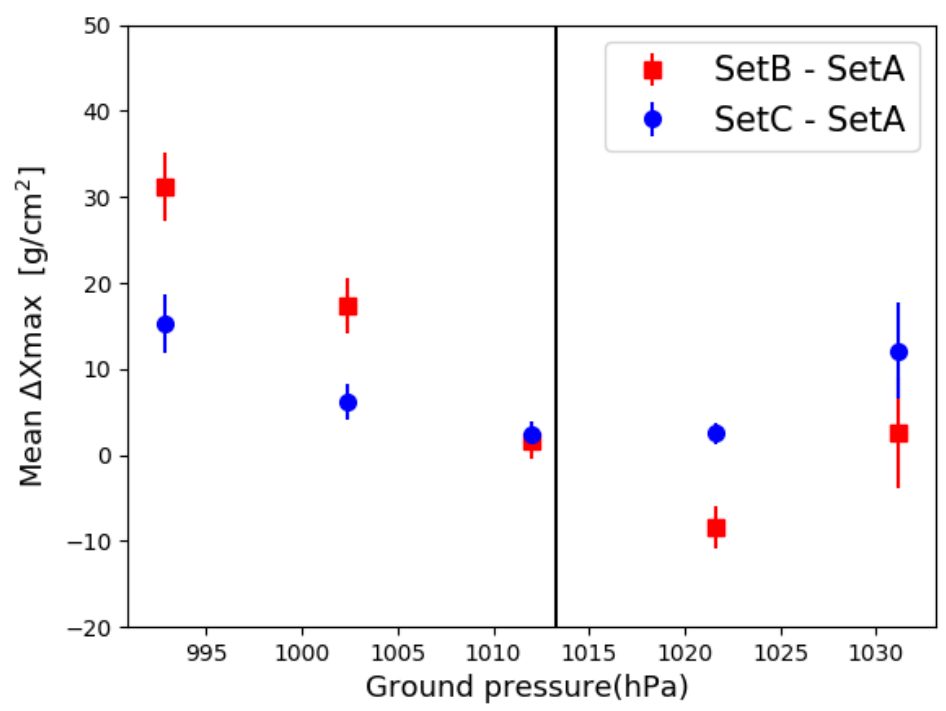

Figure 2: Difference in mean $X_{\max }$ as a function of ground pressure. The total sample contains 123 air showers recorded at LOFAR. The black line denotes the U.S standard atmospheric pressure. 
In Fig. 2 the difference in mean reconstructed $X_{\max }$ between the various simulation sets mentioned above is plotted against ground pressure obtained from GDAS. Both the blue circles and red squares converge to zero where GDAS pressure approaches the US standard pressure at 1013 $\mathrm{hPa}$. The red squares have large $\Delta \mathrm{X}_{\max }$ in general. This is expected from the fact that there is no atmospheric correction involved in Set-B. The blue circles however show a significant deviation both at low and high pressure values. This suggests that the linear first order correction added to the standard US atmosphere implemented in Set-C is not sufficient. Full GDAS-based atmospheric profiles become indispensable when the air pressure at the ground deviates by more than $10 \mathrm{hPa}$ from the US standard atmosphere value.

\subsection{Effects of humidity}

In the radio frequency regime, the humidity results in higher values of refractive index. For this study, two sets of simulations were produced. In one set the shower was simulated with a GDAS atmosphere with extreme humid weather conditions and in the other with a GDAS atmosphere with zero humidity. The same atmospheric parameters are used in both cases to ensure that the particles evolve in a similar way in the atmosphere and produce same shower maximum. In this way the inclusion of humidity only influences the simulated radio pulses. The difference in the refractive index contributes to difference in propagation effects on the pulse arrival time and power. The lateral distribution of the energy fluence, the time-integrated power per unit area, for different observer positions is also studied for different frequency bands for these two cases, as shown in Fig-3. In the low frequency band of 30-80 MHz relevant for LOFAR the difference in the fluence between the two sets is small, up to the order of $4 \%$. In the high frequency band of $50-350 \mathrm{MHz}$ the values are larger being around $10 \%$. In the higher frequency band the Cherenkov-like effects become stronger and the signal is compressed along the Cherenkov ring [7]. The opening angle is strongly dependent on the index of refraction. This explains the higher difference in power in Fig-3.

To investigate the effect of humidity on $\mathrm{X}_{\max }$ measurements we have performed a Monte Carlo comparison study between two sets of simulations that deals with the atmospheres in a similar way as described in the beginning of this section. For each of theses cases we have used a set of 40 simulated events with different energy, zenith and azimuth angles. Each of these showers have an ensemble of proton and iron initiated showers selected with CONEX. One shower from the set with realistic humidity is taken as reference and all the simulated showers from the set with zero humidity are used to perform the reconstruction. This yields a reconstructed $X_{\text {reco }}$ that can be compared to the actual $X_{\text {real }}$ of the reference shower. The same method is repeated for all the showers in the humid set. The difference $X_{\text {reco }}-X_{\text {real }}$ estimates the effect of humidity on reconstructed $X_{\max }$. Results are shown in Fig.4. We do not observe any significant shift in $X_{\max }$ in this study. This indicates that these effects are most likely smaller than the overall resolution in reconstructed $\mathrm{X}_{\max }$ in the LOFAR frequency band. We also performed the same study in a higher frequency band between 50 and $350 \mathrm{MHz}$, corresponding to the SKA-low band. There, an overall shift of $6.8 \mathrm{~g} / \mathrm{cm}^{2}$ in the reconstructed $\mathrm{X}_{\max }$ was observed. 

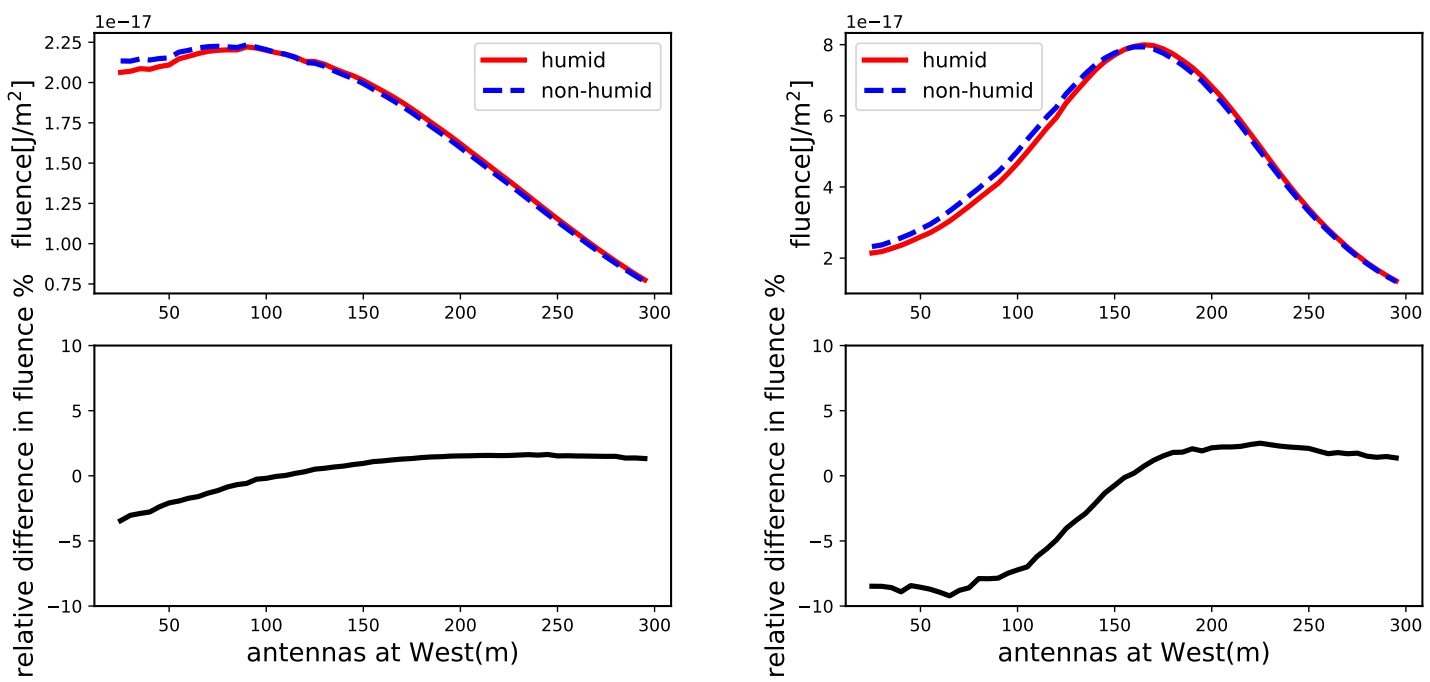

Figure 3: Radio lateral distribution profiles for a $10^{17} \mathrm{eV}$ proton shower coming from zenith $45^{\circ}$ with true $\mathrm{X}_{\max }=593 \mathrm{~g} / \mathrm{cm}^{2}$. Observers are located to the west of the shower core. Left: low frequency band between $30-80 \mathrm{MHz}$, Right: high frequency band between $50-350 \mathrm{MHz}$. The upper panel shows the LDF of total fluence for the humid and non-humid sets, the lower panel shows the relative difference between these two.
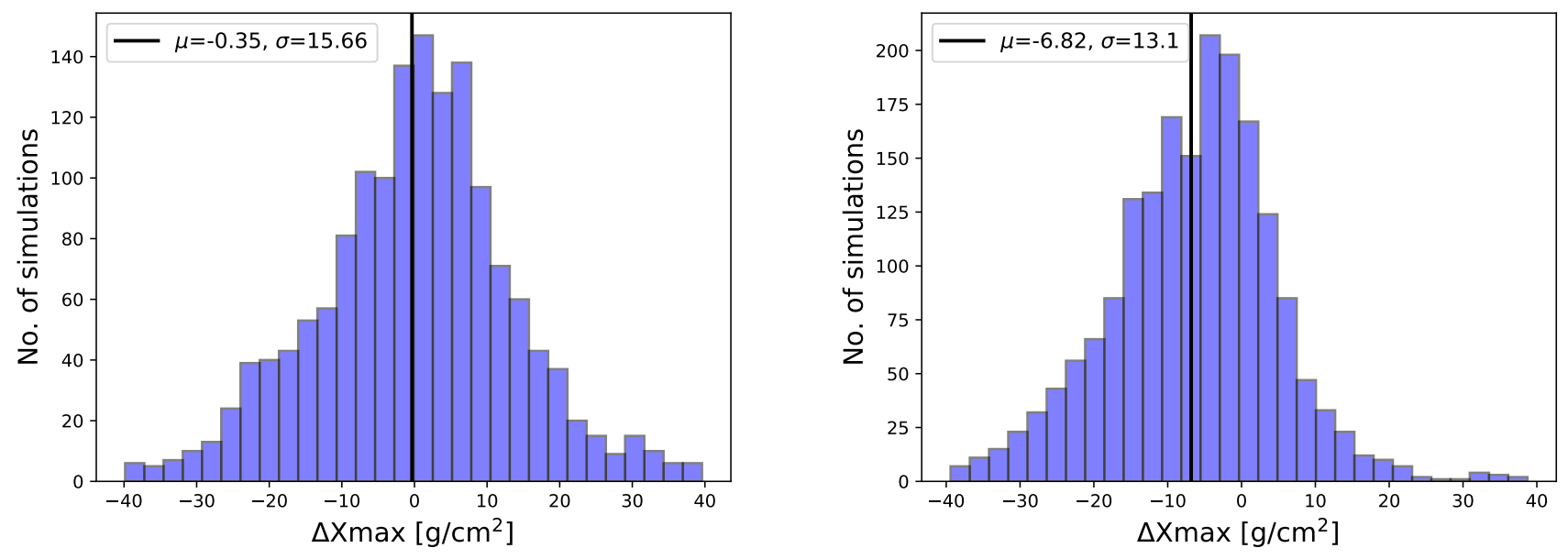

Figure 4: Histogram for the $\Delta X_{\max }=X_{\text {reco }}-X_{\text {real }}$ between the reconstructed and true value of the $X_{\max }$ obtained from the Monte Carlo study between the humid and non-humid simulation sets. Left: for the low frequency band of $30-80 \mathrm{MHz}$. Right: for the high frequency band of $50-350 \mathrm{MHz}$. The shift in the $\mathrm{X}_{\max }$ is significant at $2 \sigma$ level.

\section{Conclusions}

Atmospheric effects are important for the precise reconstruction of $X_{\max }$ with the radio technique. We have developed a tool that extracts atmospheric parameters using GDAS for a given time and location. Using this we have implemented a time-dependent parameterized density and 
refractive index profiles in CORSIKA/CoREAS. This code is flexible and can be used for other air shower experiments as well. Using LOFAR data, we demonstrated the importance of using full GDAS-based atmospheres instead of a linear geometrical correction to the US standard atmosphere. While this correction is sufficient for the bulk of the events, it becomes indispensable for extreme values for the air pressure. When the air pressure at ground level differs by less than 10 $\mathrm{hPa}$ from the US standard atmosphere value, the reconstructed $\mathrm{X}_{\max }$ value including the correction agrees to the full GDAS-based reconstruction value within $2 \mathrm{~g} / \mathrm{cm}^{2}$. However, for periods of very low air pressure, this difference grows significantly up to $15 \mathrm{~g} / \mathrm{cm}^{2}$. Effects of humidity on the energy fluence and reconstructed $\mathrm{X}_{\max }$ were probed; effects become more prominent for higher frequency bands. This could be important for the high precision $\mathrm{X}_{\max }$ measurements for the cosmic-ray detection with the SKA experiment [8].

\section{Acknowledgement}

The LOFAR cosmic-ray key science project acknowledges funding from an Advanced Grant of the European Research Council (FP/2007-2013)/ERC Grant Agreement no 227610. The project has also received funding from the European Research Council (ERC) under the European Union's Horizon 2020 research and innovation program (grant agreement No 640130). We furthermore acknowledge financial support from FOM, (FOM-project 12PR304). AN is supported by the DFG (Emmy-Noether grant NE 2031/2-1 ). LOFAR, the Low Frequency Array designed and constructed by ASTRON, has facilities in several countries, that are owned by various parties (each with their own funding sources), and that are collectively operated by the International LOFAR Telescope foundation under a joint scientific policy. We sincerely thank the CORSIKA developers for their assistance regarding the implementation of our work in CORSIKA modules.

\section{References}

[1] S. Buitink et al. A large light-mass component of cosmic rays at $10^{17}-10^{17.5} \mathrm{eV}$ from radio observations. Nature, 531:70, 2016.

[2] S. Buitink et al. Method for high precision reconstruction of air shower $X_{\max }$ using two-dimensional radio intensity profiles. Phys. Rev. D, 90(8), 2014.

[3] M. Ludwig et al. Comparison of LOPES measurements with CoREAS and REAS 3.11 simulations. AIP Conf. Proc., 1535(1):84-88, 2013.

[4] D. Heck et al. CORSIKA: A Monte Carlo code to simulate extensive air showers. Report FZKA, 6019, 1998.

[5] A. Corstanje et al. The effect of the atmospheric refractive index on the radio signal of extensive air showers. Astropart. Phys., 89:23-29, 2017.

[6] National oceanic and atmospheric administration, global data assimilation system. https://www.ncdc.noaa.gov/data-access/model-data/model-datasets/global-data-assimilation-systemgdas.

[7] A. Nelles et al. A parameterization for the radio emission of air showers as predicted by CoREAS simulations and applied to LOFAR measurements. Astropart.Phys., 60:13-24, 2015. 
[8] T. Huege et al. High-precision measurements of extensive air showers with the SKA. PoS, ICRC2015:309, 2016. 